\title{
Dynamical properties of myoglobin in an ultraviscous water-glycerol solvent investigated with elastic neutron scattering and FTIR spectroscopy
}

\author{
Fabio Librizzi ${ }^{\mathrm{a}, *}$, Antonino Caliò ${ }^{\mathrm{b}}$, Antonio Cupane ${ }^{\mathrm{b}}$ \\ a Institute of Biophysics, National Research Council, Via Ugo La Malfa 153, 90146 Palermo, Italy \\ ${ }^{\mathrm{b}}$ Department of Physics and Chemistry, University of Palermo, Viale delle Scienze Ed. 18, 90123 Palermo, Italy
}

\section{A R T I C L E I N F O}

\section{Article history:}

Received 14 April 2018

Received in revised form 3 July 2018

Accepted 13 July 2018

Available online $\mathrm{xxxx}$

\section{Keywords:}

Protein dynamical transition

Neutron scattering

Mean square displacements

Fourier transform infrared spectroscopy

Amide bands

\begin{abstract}
A B S T R A C T
Proteins have distinctive dynamical properties, characterized by the fluctuations of protein molecules among the different minima of their energy landscape. These fluctuations, progressively activated for temperature values larger than $\sim 180 \mathrm{~K}$, lead to a steep increase in the temperature dependence of all measurable dynamical properties. This phenomenon is known as Protein Dynamical Transition and, in spite of the intense studies due to its importance in protein function and to the relation with the fascinating fundamental thermodynamics of complex systems, many aspects of it are not yet clearly understood. Among these, the relationship with the properties of the external solvent and the molecular details of the involved protein motions still need further investigations. We report here a comparative study of the Dynamical Transition in a Protein-Glycerol-Water system, from two different points of view: i) Elastic Neutron Scattering (ENS), which gives the Mean Square Displacements of the hydrogen atoms of the protein and is particularly sensitive to side chain motions; ii) Fourier Transform Infrared Spectroscopy (FTIR) in the Amide regions, which is sensitive mainly to the properties of the backbone atoms of the protein. The obtained results show an almost superimposable thermal behavior of protein backbone (FTIR data) and side chains (ENS data). Thus, in our experimental conditions, the Protein Dynamical Transition emerges as a unique thermodynamic process related to the properties of the external Glycerol/Water medium and implying a general softening of the whole protein molecule (backbone and side chains), which is a prerequisite for protein function.
\end{abstract}

(c) 2018 Elsevier B.V. All rights reserved.

\section{Introduction}

The dynamical properties of proteins are characteristically different at low and high temperatures. At low temperature (below $~ 180 \mathrm{~K}$ ) proteins behave like other solid systems and harmonic motions dominate. On the contrary, above $\sim 180 \mathrm{~K}$ protein internal dynamics is characterized by strong non-harmonic contributions, which are believed to be functionally important, since they provide the flexibility necessary for any function [1-3]. Given their importance, these additional motions have been widely investigated over the last 3 decades by a number of experimental techniques, such as Neutron Scattering [4-7], Mössbauer Spectroscopy [8], X-Rays and Neutron Crystallography [9, 10] and many other spectroscopic methods [11-13]. These intense efforts allowed to highlight the relationship between the dynamical properties of proteins and their thermodynamic complexity, which can be well described in terms of a multiminima energy landscape (for a deeper discussion see e.g. Refs. [7, 14]). In facts, proteins may exist in a very

\footnotetext{
* Corresponding author.

E-mail address: fabio.librizzi@pa.ibf.cnr.it (F. Librizzi).
}

large number of slightly different configurations, known as Conformational Substates [1-3]. At low temperature, each protein molecule is frozen in one particular conformational substate, and therefore the system can be described in terms of harmonic potentials. As the temperature increases, new contributions to the dynamics can be triggered. Firstly, the activation of methyl group rotations can be observed [15, 16], at temperature values varying from $\sim 100$ to $\sim 180 \mathrm{~K}$, depending on the experimental time resolution [7]. Further increasing the temperature, protein molecules start to fluctuate among different conformational substates, and these fluctuations bring about a steep increase in all measured dynamical properties of the system. This phenomenon, i.e. the crossover observed at $\sim 180 \mathrm{~K}$ in the thermal behavior of protein dynamics, is usually referred to as Protein Dynamical Transition (PDT) [4]. It is still extensively studied because many aspects are not yet clearly understood, as for instance its dependence on the properties of hydration water [17-21], on the characteristics of the external medium in general [22-25], and on other thermodynamic variables such as pressure [26-28].

As mentioned before, Neutron Scattering is one of the most powerful techniques for the investigation of PDT. In particular, Elastic Neutron 
Scattering (ENS) is widely used for its ability to furnish the Mean Square Displacements (MSD) of protein non-exchangeable hydrogen atoms, whose incoherent scattering, due to their very large incoherent cross section, dominates the measured signal $[29,30]$. Since hydrogen atoms are evenly distributed in proteins, a clear distinction between side chains and backbone contributions to the observed dynamics is rather difficult by using ENS alone, depending on instrumental resolution, solvent composition and temperature. However, it has been suggested that the measured MSD of hydrogen atoms in proteins is largely due to side-chain motions, with only small contributions from the backbone of the protein [10]. In view of the above considerations, it would be highly desirable to investigate the PDT on suitable protein samples with the aim of comparing the thermal dependence of the MSD of non-exchangeable hydrogen atoms, as obtained by ENS, with other measurable quantities related to the dynamic properties of backbone atoms. This can be of particular interest also in view of the fact that it has been very recently reported that protein backbone atoms may display different dynamical properties with respect to side chains hydrogen atoms [31].

A suitable tool to probe the properties of backbone atoms in proteins can be Fourier Transform Infrared (FTIR) Spectroscopy, in the region of the Amide bands. In fact, as known, these bands are associated to vibrations involving the backbone atoms of the proteins, and are particularly sensitive, especially in the case of Amide I', to the conformational changes of the protein, since different secondary structure elements give rise to different absorptions contributions [32-35].

In view of the above considerations, we have studied the thermal dependence of the Amide I' and Amide II' bands of myoglobin in the temperature range 20-300 Kand compared it with the thermal dependence of hydrogen atoms MSD measured in an identical sample with ENS [26]. Aim of the study is to compare the dynamic properties (in particular concerning the PDT) of the protein backbone with those of the protein amino acid side chains. The composition of the sample (Myoglobin $50 \%, D_{8}$-Glycerol 33.5\%, $D_{2} 016.5 \%$, weight fractions) was chosen with the dual purpose of ensuring a good signal to noise ratio in experiments at cryogenic temperatures and a protein hydration level sufficient for the activation of the PDT and therefore of functionally important motions [17, 36, 37]. Previous studies on the dynamical properties of proteins in mixtures of water and glycerol $[38,39]$, or even in ternary mixtures with trehalose $[40,41]$ have shown that protein internal dynamics is strongly coupled to the properties of the external medium. Moreover, interesting correlations between the effects of the external medium on the internal dynamics and on the protein melting temperature have been reported [42].

\section{Materials and methods}

\subsection{Sample preparation}

Myoglobin (from horse skeletal muscle) and $\mathrm{D}_{2} \mathrm{O}$ were purchased from Sigma-Aldrich and used without further purification. $\mathrm{D}_{8}$-Glycerol was purchased from Cortecnet Europe (Voisins-Le-Bretonneux, France). Myoglobin was first dissolved in $\mathrm{D}_{2} \mathrm{O}$ to allow the deuterium exchange of labile hydrogens, and then lyophilized after two days equilibration at room temperature. Then, following a previously reported procedure $[26,43]$, the sample was prepared by successive slow additions of deuterated protein (up to $400 \mathrm{mg}$ ) in $400 \mathrm{mg}$ of solvent (66\% $\mathrm{D}_{8}$-Glycerol $-33 \% \mathrm{D}_{2} \mathrm{O}$ ). Further addition of small amounts $\mathrm{D}_{2} \mathrm{O}$ was necessary to facilitate protein dissolution and excess $\mathrm{D}_{2} \mathrm{O}$ was removed by nitrogen flux upon the sample, until the desired weight proportions were reached: Myoglobin 50\%, $\mathrm{D}_{8}$-Glycerol 33.5\%, $\mathrm{D}_{2} 0$ 16.5\%.

\subsection{Neutron scattering measurements}

Elastic Neutron Scattering (ENS) measurements were performed at ILL (Grenoble, France) using the backscattering spectrometer IN13.
The experimental setup and procedures and data reduction are described in ref. [26]. The total MSD here reported were obtained in the framework of the Gaussian approximation [29], by considering, for each temperature value, a suitable q-range, obtained by fixing $q_{\max }$ at the maximum value which consistently satisfies the condition $\left(6\left\langle\Delta x^{2}\right\rangle\right.$ tot $\left.\cdot q_{\max }^{2}\right)<2$ [44-46]. This allows to maximize the q-range used in each linear fit and therefore to minimize the error in the obtained parameters, by means of an unbiased automatized procedure [26].

\subsection{FTIR measurements}

Infrared measurements were carried out on a Jasco FT/IR-410 Spectrometer. A $25 \mu \mathrm{m}$ teflon spacer was used, between two $\mathrm{CaF}_{2}$ windows. Temperature in the range $20-300 \mathrm{~K}$ was controlled by a helium closed cycle cryostat (Oxford Instruments), operating in conjunction with a Cryodrive 3.0 compressor.

The spectrometer chamber was continuously purged with a nitrogen flux, in order to eliminate contributions from the atmosphere. Measurements were performed from low to high temperature, allowing $25 \mathrm{~min}$ of thermalization before the recording of each spectrum. A solvent spectrum was also recorded at room temperature. Resolution was set at $1 \mathrm{~cm}^{-1}$ and 256 acquisitions were averaged for each temperature. In the spectral region of interest ( $1300-1755 \mathrm{~cm}^{-1}$ ) solvent absorption resulted to be very low. In order to minimize the noise due to imperfect purging of the spectrometer chamber, the solvent spectrum was subtracted from all sample spectra multiplying it by a constant $k$, suitably determined from the minimization of the square of the 2nd derivative of the resulting spectrum.

Spectra were then analyzed by curve fitting in the Amide $\mathrm{I}^{\prime}$ and Amide II' regions. For both bands, a straight baseline and two Gaussian peaks were used. To minimize noise in the obtained results, parameters showing a small and not definite temperature behavior were held constant for all temperature values.

\section{Results and discussion}

Fig. 1 reports the MSD as measured by ENS for Mb in our conditions (protein-glycerol-water mixture) [26] and, for the sake of comparison, in standard hydrated $\mathrm{Mb}$ powders [4]. As evident, in both cases, for temperatures above $\sim 180 \mathrm{~K}$, MSD values display a nonlinear increase, indicating the onset of the non-harmonic motions typical of PDT and arising from the fluctuations of each protein molecule among its different conformational substates [1-3]. Although extracted from experimental data by following two different methods (namely, Gaussian Approximation [29, 44-46] in our case [26] and double-well model for

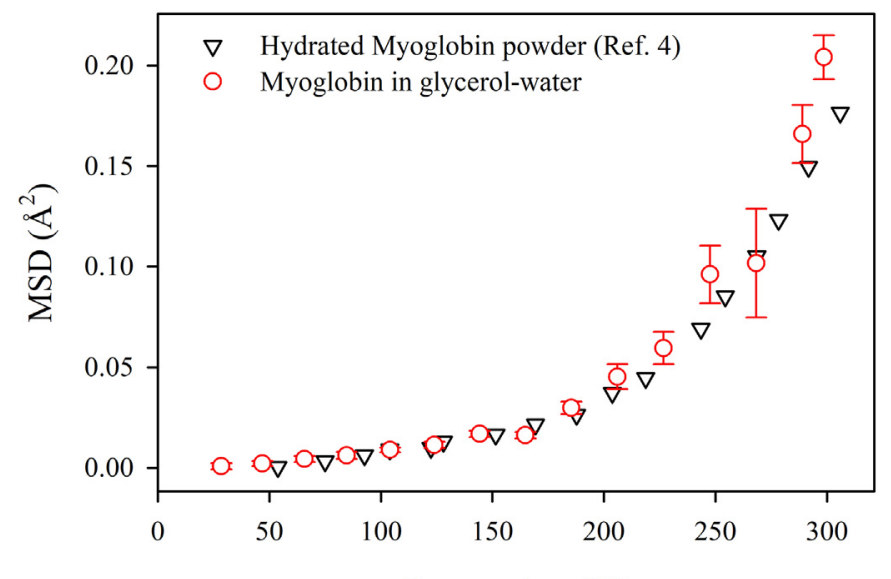

Temperature $(\mathrm{K})$

Fig. 1. MSD for myoglobin in our experimental conditions (Ref. 26) and in hydrated powders (Ref. 4). 
ref. [4]) the MSD reported in Fig. 1 are in both cases the total MSD within the accessible time window of the instrument, which is the same for the two experiments. Therefore, the good agreement between the two sets of data indicates that our experimental conditions are suitable for the investigation of protein internal dynamics.

Fig. 2a shows the normalized FTIR absorption spectra for our sample at different representative temperatures, in the region of the Amide I' band. The main effect of increasing temperature is a slight transfer of the absorption signal from the low- to the high-wavenumber region. This effect appears much stronger in the temperature interval 180$290 \mathrm{~K}$ than in the temperature interval $20-180 \mathrm{~K}$. A simple method to extract the overall temperature dependence of the spectra shown in Fig. 2a, not biased by any model, can be the calculation of the their 1st moment (i.e. the average frequency). This parameter is reported in Fig. 2b (black circles, left axis), together with the MSD values obtained by ENS for an analogous sample. The almost exact coincidence of the two data sets is a new and interesting result since the two experimental techniques measure completely different features: FTIR spectroscopy deals with equilibrium spectral properties of atoms mainly localized on the peptide bonds of the protein backbone, while neutron scattering gives the dynamics (in the time window of $\sim 100 \mathrm{ps}$ ) of the nonexchangeable hydrogen atoms, evenly distributed throughout the protein structure and mainly localized in the amino-acid side-chains. In spite of the diversity of the two techniques, the overall temperature dependence of FTIR spectra, as rendered by their average frequency, results quite similar to the temperature behavior observed by neutron

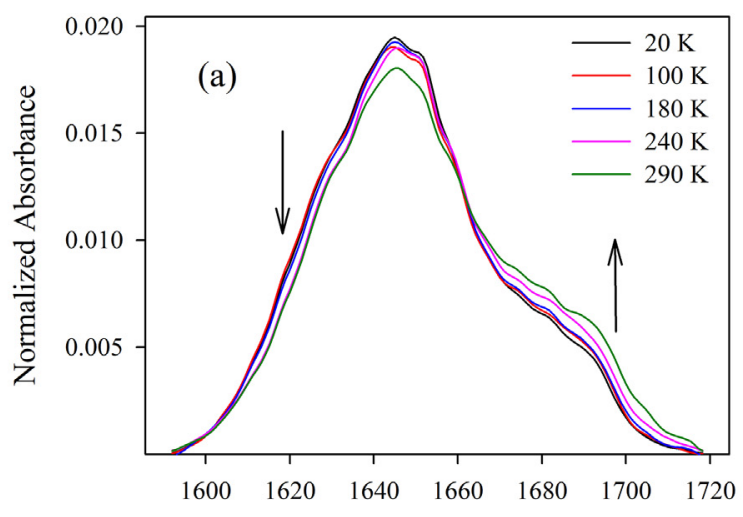

Wavenumber $\left(\mathrm{cm}^{-1}\right)$

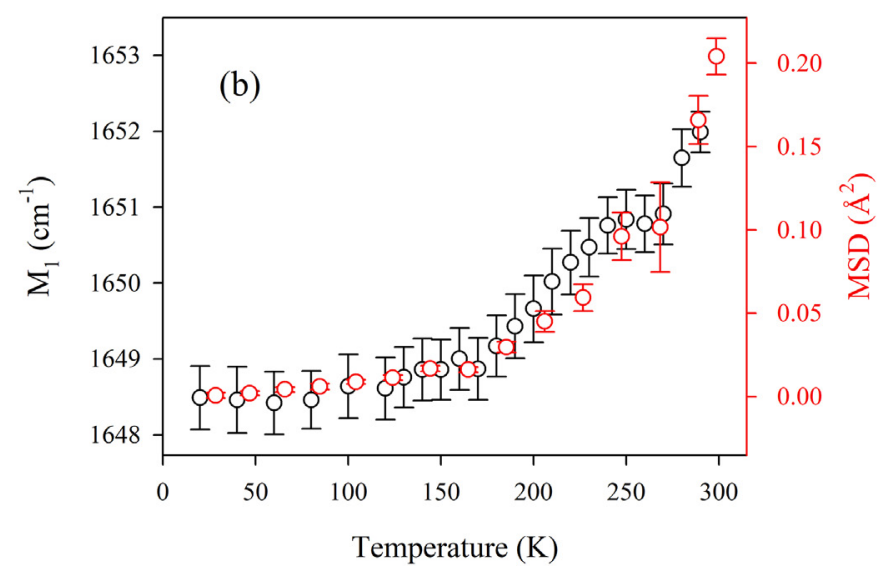

Fig. 2. (a) Normalized FTIR spectra relative to the amide I' band at different temperatures. The arrows indicate the changes observed at increasing temperature values. (b) 1 st moment of the absorption bands shown in (a), as a function of temperature (black circles, left y-axis), and MSD obtained by neutron scattering for the analogous MbGlycerol-water sample (red circles, right axis). (For interpretation of the references to colour in this figure legend, the reader is referred to the web version of this article.) scattering, with more pronounced changes in the measured values for temperatures above $\sim 180 \mathrm{~K}$. Most likely, for both techniques, the drastic regime change observed at $\sim 180 \mathrm{~K}$ must be related to the PDT.

A more detailed analysis of the Amide I' band can give useful insights into the thermal dependence of FTIR spectra shown in Fig. 2. As known, Amide $\mathrm{I}^{\prime}$ band is mainly associated with the $\mathrm{C}=0$ stretching vibration of the peptide group and strongly depends on the secondary structure of the investigated protein [32-34]. Here, we analyzed the Amide I' band of myoglobin in terms of two Gaussian absorption sub-bands, as shown in Fig. 3 for the sample at $20 \mathrm{~K}$. From now on, we will designate these two Gaussian bands as $\mathrm{A}_{1}\left(\sim 1645 \mathrm{~cm}^{-1}\right)$ and $\mathrm{A}_{2}\left(\sim 1686 \mathrm{~cm}^{-1}\right)$. Even if more elaborate analyses by using a larger number of peaks are possible and in some cases more appropriate [47], we prefer here to take into account only two main contributions to the overall band, which can be easily attributed to its principal secondary structure elements: $\alpha$-helices $\left(A_{1}\right)$ and turns and/or disordered structures $\left(A_{2}\right)[32$, 33,48 ] (we recall that no sizeable $\beta$ structure is present in myoglobin).

Fig. 4 a reports the ratio $A_{2} / A_{1}$ between the areas of the two Gaussian bands as a function of temperature (black circles, left axis); as in Fig. 2a, the MSD values obtained by ENS for an analogous sample are also reported. Data in Fig. 4a clearly show that the $M_{1}$ shift (see Fig. $2 b$ ) is due to an $A_{1} \rightarrow A_{2}$ intensity transfer rather than to a frequency shift of both sub-bands. Consistently, the temperature dependence of the frequency of the two sub-bands was found to be not significant (within $\pm 2 \mathrm{~cm}^{-1}$ ) and fittings of excellent quality could be obtained also by keeping their values temperature independent (data not shown). At variance, in previous reports on the thermal behavior of the Amide I' band in proteins at cryogenic temperatures, the observed temperature induced spectral variations were represented mainly in terms of frequency shifts [49-51]. Instead, in model peptide systems, a temperature dependence of relative contributions of different sub-bands has been reported [51].

Fig. $4 \mathrm{~b}$ reports the temperature dependence of bandwidths: at high temperature we observe an increase in the $A_{2}$ width $\left(\sigma_{2}\right)$ and a small, barely detectable, decrease in the $A_{1}$ width $\left(\sigma_{1}\right)$. The increase in $\sigma_{2}$ can be rationalized in terms of a corresponding increase in the conformational heterogeneity brought about by high temperature. In this sense, the small decrease in $\sigma_{1}$ is somewhat counterintuitive. It must be noted, however, that in some cases the width of infrared bands may decrease at high temperature [52]. This phenomenon, referred to as motional narrowing, may arise from the fast conformational averaging of protein groups and solvent molecules around the infrared absorber [33, 53-55]. In the case of $A_{1}$ in the Amide $I^{\prime}$ band (Fig. $4 b$ ), the narrowing seems to be quite small, barely above our experimental

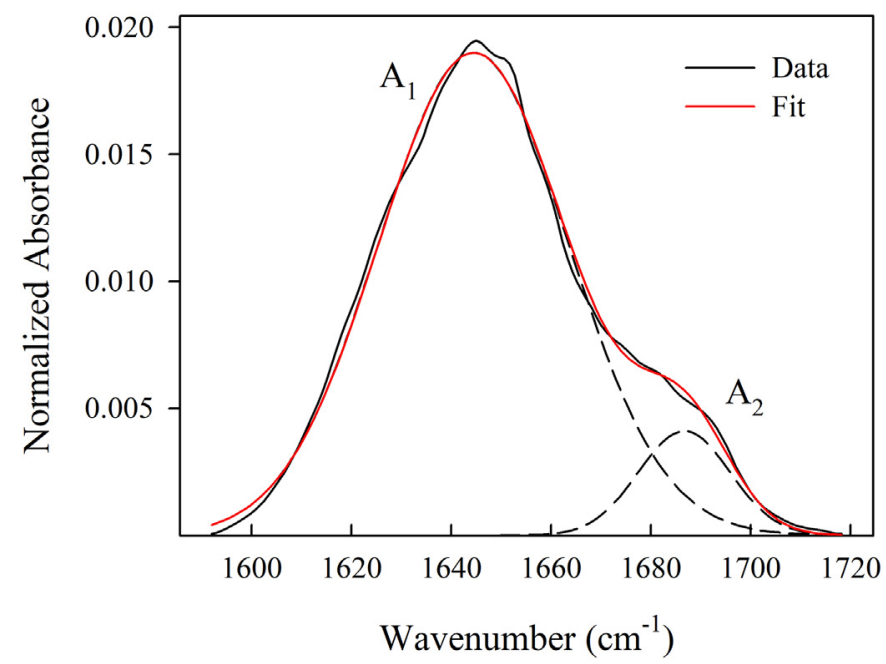

Fig. 3. Analysis of the Amide $\mathrm{I}^{\prime}$ band for the sample at $20 \mathrm{~K}$, in terms of two Gaussian subbands. 


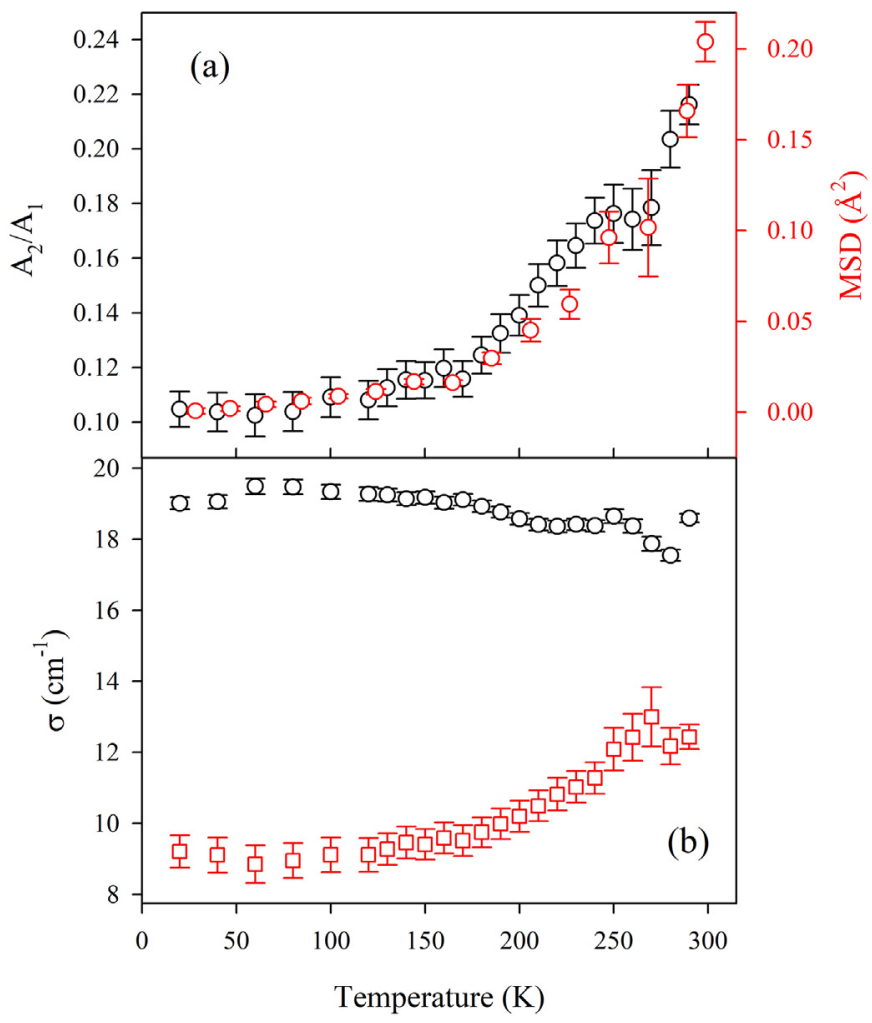

Fig. 4. (a) Ratio $A_{2} / A_{1}$ between the areas of the two Gaussian bands in Amide I' as a function of temperature (black circles, left axis); for the sake of comparison, data are superimposed to the MSD obtained by ENS on an analogous sample (red circles, right axis). (b) Gaussian width $\sigma$ of the two bands, $A_{1}$ (black circles) and $A_{2}$ (red squares). (For interpretation of the references to colour in this figure legend, the reader is referred to the web version of this article.)

resolution. We will see in the following that this effect is much more pronounced for the Amide II' band.

The close similarity between the temperature dependence of FTIR and ENS data reported in Figs. 2b and $4 \mathrm{a}$ is not trivial and deserves some further comments. As mentioned in the Introduction, it has been reported that mainly the side-chain hydrogen atoms contribute to the MSD measured by ENS in the 100 ps time scale [10]. On this basis, a comparison of ENS data with techniques able to probe the structural and dynamical properties of protein's backbone atoms, as FTIR in the amide region, is highly desirable also in view of very recent results indicating that in some cases the backbone atoms may behave differently from hydrogen atoms of the side chains, with respect to the PDT. In particular, in extremely dry proteins heavy backbone atoms seem to display a dynamical transition at $180-200 \mathrm{~K}$, which is usually absent when non-exchangeable hydrogen atoms are the main contributors to the observed signal [31]. In fact, in this last case, a much larger hydration ( $h \sim 0.1-0.2$ ) is required to observe the dynamical transition [17]. In this framework, the above reported analogous temperature dependence of ENS and FTIR data (Figs. 2 and 4) may furnish important indications. The two techniques give two complementary points of view of the dynamical transition in our system. PDT arises from the thermal fluctuations of each protein molecules among the very large number of minima in its conformational energy landscape [1]. These fluctuations bring about a steep increase in the MSD of the non-exchangeable hydrogen atoms of the system, mainly located at the amino acid $\alpha$ carbons and side chains, as revealed by neutron scattering. On the other hand, FTIR data indicate that, concerning the protein backbone atoms probed by the Amide I' band, at temperatures larger than $\sim 180 \mathrm{~K}$ a general softening of the protein occurs, associated to an increase in the portion of the protein involved in turns and/or disordered regions.
Useful information can be obtained also from the analysis of the Amide II' $^{\prime}$ band. Fig. 5a reports the normalized absorption spectra of our sample in the region of the Amide II' band, for some representative temperature values. Notwithstanding the larger noise in the data, the spectra clearly show that Amide II' band is more sensitive to temperature with respect to Amide I' (Fig. 2) and that, as for Amide I', the majority of the changes are detected for temperatures above $180 \mathrm{~K}$. Again, as previously done for Amide I', the 1 st moment (the average frequency) of the spectra shown in Fig. 5a can be used as an unbiased parameter to visualize the overall effects of temperature increase. As shown in Fig. 5b, also in this case the thermal behavior is analogous to the one observed for the MSD of the hydrogen atoms obtained by ENS (note that in Fig. $5 b$, for the sake of comparison, the left $y$-axis, relative to $M_{1}$ has been reverted).

Here also, we analyzed the absorption profile by means of two Gaussian bands (see Fig. 6), denoted as $B_{1}(\sim 1400 \mathrm{~cm}-1)$ and $B_{2}$ $(\sim 1445 \mathrm{~cm}-1)$. We stress that, even if in some cases Amide II' band is used as an empirical tool to monitor protein secondary structure [56, 57], no clear attribution to specific secondary structure elements is usually performed. Some of the parameters obtained by this analysis are reported in Fig. 7. As for Amide I', the ratio between the areas of the two sub-bands (Fig. 7a) shows a temperature dependence analogous to what observed by ENS for the hydrogen atoms MSD. At difference from Amide I', we observe in Amide II' a sizeable red shift of the peak frequency (Fig. 7b) with increasing temperature, both for $B_{1}$ and $B_{2}$. A
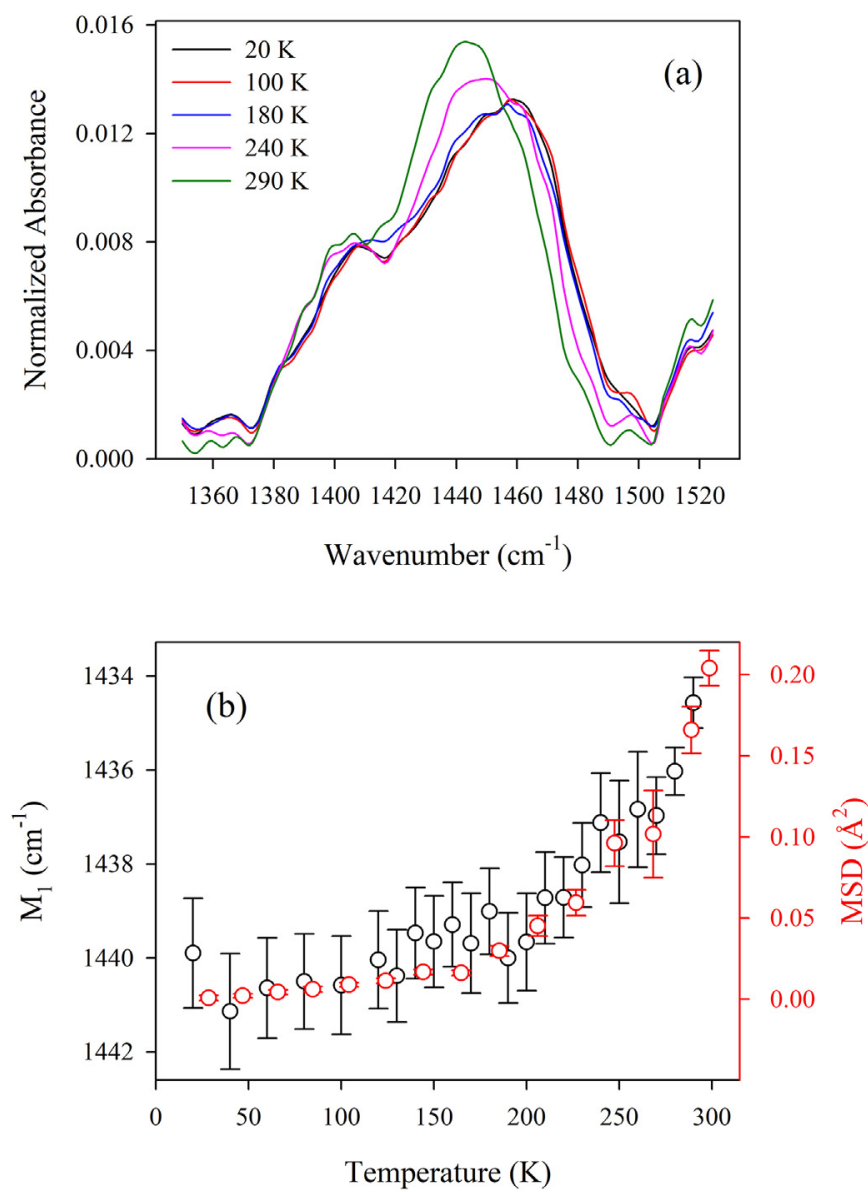

Fig. 5. (a) Normalized FTIR spectra relative to the amide II' band at different temperatures. (b) 1 st moment of the absorption bands shown in (a), as a function of temperature (black circles, left y-axis), and MSD obtained by neutron scattering for the analogous MbGlycerol-water sample (red circles, right axis). For the sake of comparison, the left y-axis is reversed. (For interpretation of the references to colour in this figure legend, the reader is referred to the web version of this article.) 


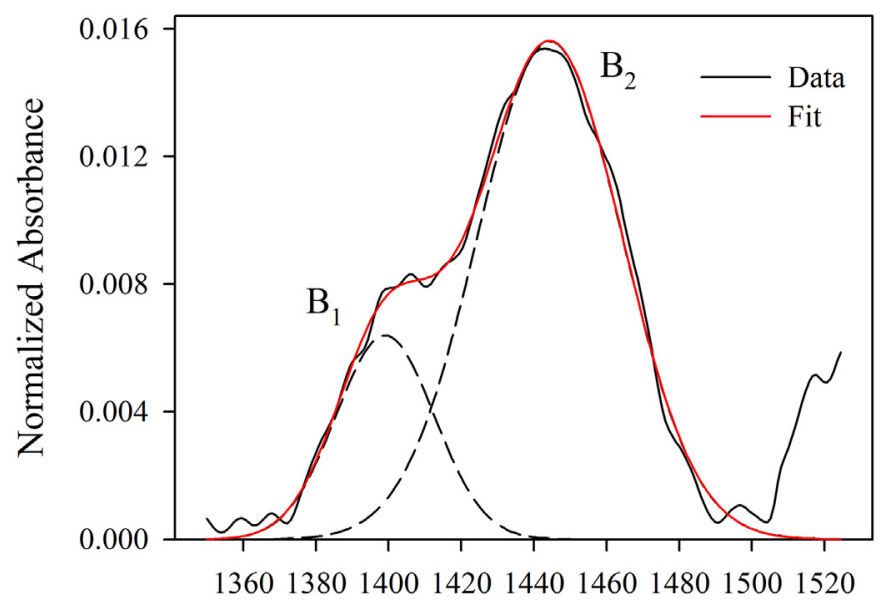

Wavenumber $\left(\mathrm{cm}^{-1}\right)$

Fig. 6. Analysis of the Amide II' band for the sample at $290 \mathrm{~K}$, in terms of two Gaussian subbands.

similar frequency red shift has been reported in ref. [49] for the amide II band of myoglobin in Glycerol/ $\mathrm{H}_{2} \mathrm{O}$.

We also observe a strong reduction with temperature in the Gaussian width of $B_{1}$, occurring above $180 \mathrm{~K}$ (Fig. 7c), while the same parameter for $B_{2}$ is found essentially temperature independent (data not shown), and therefore was held constant in the fitting procedure at the value of $20 \mathrm{~cm}^{-1}$. As mentioned before, the counterintuitive decrease in the $\mathrm{B}_{1}$ Gaussian width may be rationalized in terms of motional narrowing, arising from the fast conformational averaging of protein groups and solvent molecules around the infrared absorber [33, 52-55].

Finally, Fig. 8 shows a Van't Hoff plot of the ratio between the areas of the two Gaussian bands obtained in the analysis, both of the Amide $\mathrm{I}^{\prime}$ (black circles) and Amide II' (blue square). As shown by the straight lines in the figure, the data points follow an Arrhenius behavior for temperature values above $\sim 180 \mathrm{~K}$, while they are almost constant at lower temperatures, indicating that below $\sim 180 \mathrm{~K}$ (i.e. below the glass transition of the water/glycerol solvent) the equilibrium between $A_{1}\left(B_{1}\right)$ and $A_{2}\left(B_{2}\right)$ populations is frozen. Interestingly, the two $\Delta H$ values obtained in the Arrhenius region are compatible within the experimental uncertainties, being $(1.9 \pm 0.1) \mathrm{kJ} / \mathrm{mol}$ in the case of Amide $\mathrm{I}^{\prime}$ and $(2.1 \pm 0.3)$ $\mathrm{kJ} / \mathrm{mol}$ for Amide II'; this suggests, for $\mathrm{B}_{1}$ and $\mathrm{B}_{2}$, a spectral attribution analogous to that given for $A_{1}$ and $A_{2}$.

\section{Conclusions}

In this work we have used temperature dependent IR absorption and elastic neutron scattering to characterize the dynamical properties of myoglobin in an ultraviscous $\mathrm{D}_{2} \mathrm{O} /$ Glycerol environment. Since IR ab-

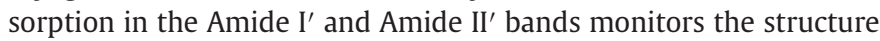
and dynamics of the protein backbone and its environment, from IR data the following scenario emerges. At temperatures lower than $\sim 180 \mathrm{~K}$ the protein/solvent system is "frozen" and no spectral variations are observed. Above $\sim 180 \mathrm{~K}$, likely in connection with the solvent glass transition, slightly lower in our conditions ( $170 \mathrm{~K})$ [58], motions of the protein backbone are allowed and one observes interconversion between different conformations; in particular, the population of proteins characterized by a looser structure with increased turns and/or disordered regions and decreased $\alpha$-helical regions increases with increasing temperature. On the other hand, our ENS measurements monitor the Mean Square Displacements of the non-exchangeable hydrogen atoms that are evenly distributed throughout the protein structure and mainly located at the amino acid $\alpha$ carbons and side chains. The MSD temperature dependence exhibits the well known dynamic activation (the so-

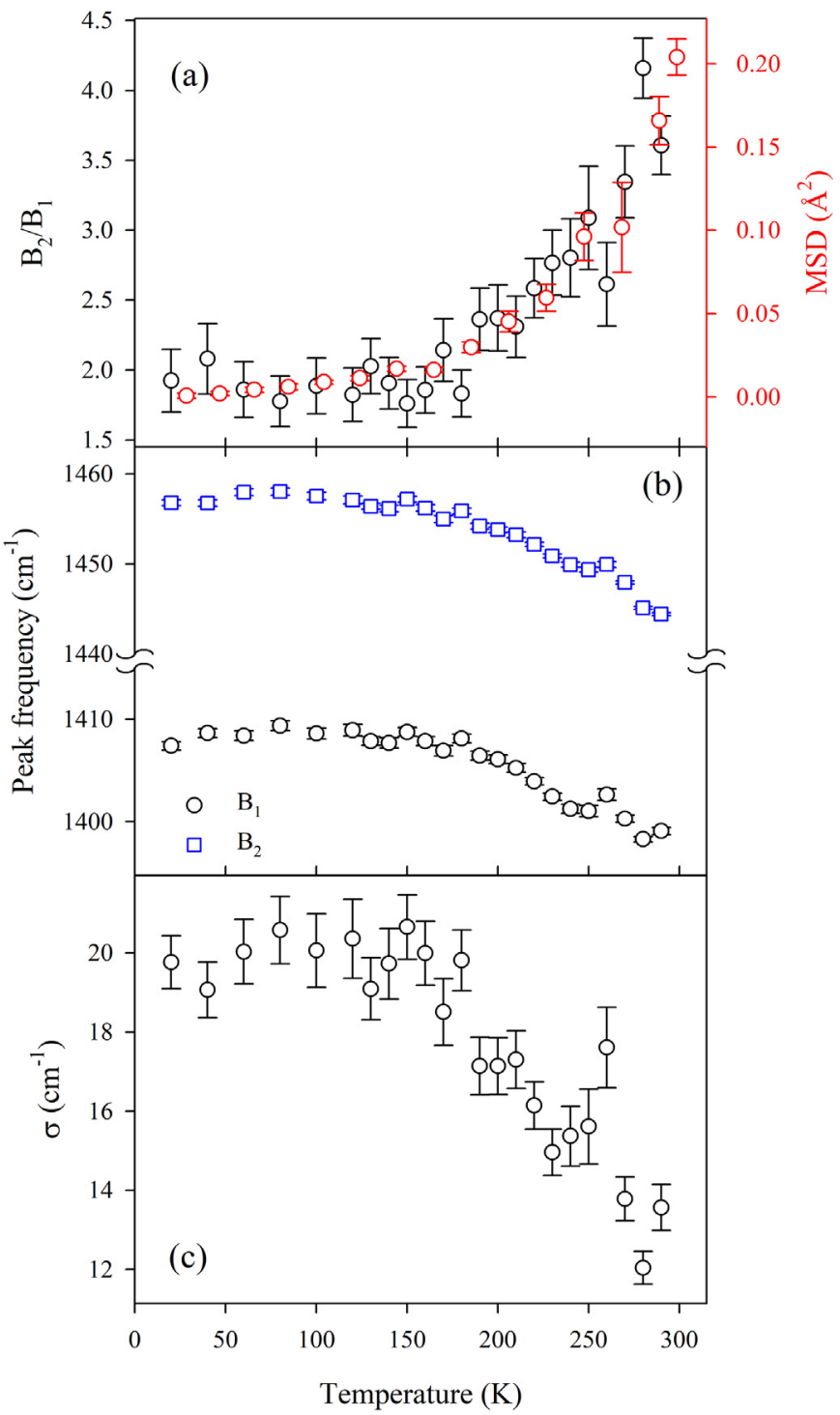

Fig. 7. (a) Ratio $B_{2} / B_{1}$ between the areas of the two Gaussian bands in Amide II' as a function of temperature (black circles, left axis); for the sake of comparison, data are superimposed to the MSD obtained by ENS on an analogous sample (red circles, right axis). (b) Peak frequency of the Gaussian peaks. (c) Gaussian width $\sigma$ the $B_{1}$ sub-band (the value for $B_{2}$ was found temperature independent and was held constant at a value of $20 \mathrm{~cm}^{-1}$ ). (For interpretation of the references to colour in this figure legend, the reader is referred to the web version of this article.)

called Protein Dynamic Transition) [4] attributed to the interconversion between statistical substates $[1,2]$ of the protein and whose onset temperature, although being an highly debated issue $[5,7,59]$, is surely located at temperatures above $180 \mathrm{~K}$. For ENS however, data the interpretation is complicated by the concomitant presence of the activation of methyl groups' rotations.

The main result of our work is that, in our experimental conditions, the two phenomena (i.e. the structural dynamics of the protein backbone and the fluctuations of the amino acid side chains, including methyl groups rotation) appear strictly coupled, as shown by the data reported in Figs. 2b, 4a, 5b and 7a. We suggest that this is due to the fact that both phenomena are mediated/determined by the external medium. Future work will verify whether this is a general conclusion or if different experimental conditions (e.g. extremely dry samples) can be found in which a decoupling is observed, as recently suggested [31]. 

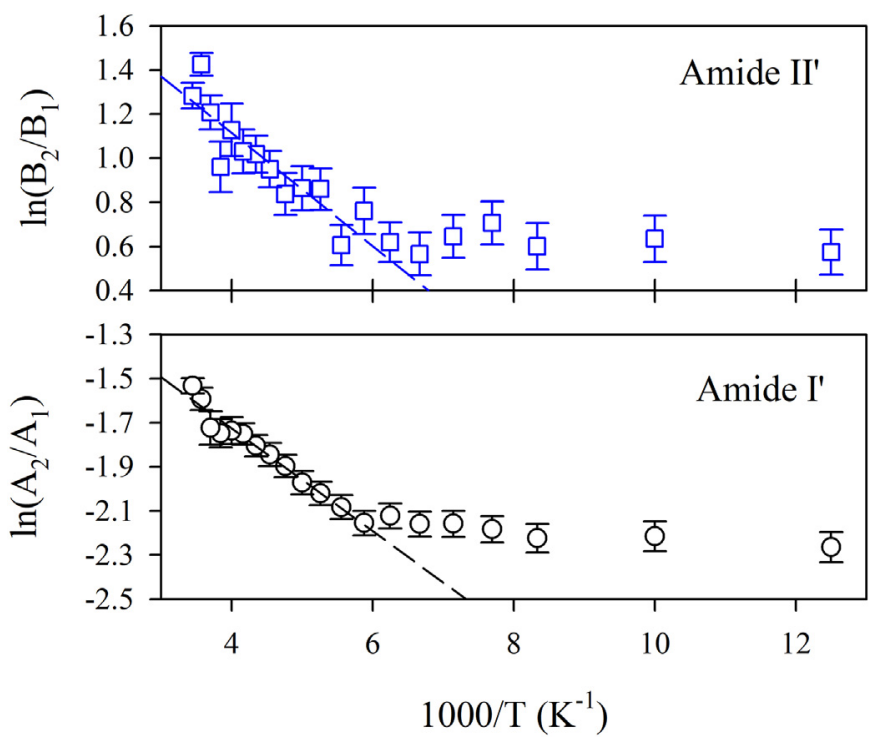

Fig. 8. Van't Hoff plot of the ratio between the areas of the two bands in Amide I' (A2/A1, black circles) and in Amide II' (B2/B1, blue squares). The straight lines show that in both cases an Arrhenius behavior is observed only for temperature values above $\sim 180 \mathrm{~K}$. (For interpretation of the references to colour in this figure legend, the reader is referred to the web version of this article.

\section{Acknowledgements}

We thank Rita Carrotta for very useful discussions and Mario Lapis and Fabrizio Giambertone for their technical support.

\section{References}

[1] H. Frauenfelder, F. Parak, R.D. Young, Conformational substates in proteins, Annu. Rev. Biophys. Biophys. Chem. 17 (1988) 451-479.

[2] H. Frauenfelder, G. Chen, J. Berendzen, P.W. Fenimore, H. Jansson, B.H. McMahon, I.R. Stroe, J. Swenson, R.D. Young, A unified model of protein dynamics, Proc. Natl. Acad. Sci. U. S. A. 106 (2009) 5129-5134.

[3] H. Frauenfelder, R.D. Young, P.W. Fenimore, Dynamics and the free-energy landscape of proteins, explored with the Mössbauer effect and quasi-elastic neutron scattering, J. Phys. Chem. B 117 (2013) 13301-13307.

[4] W. Doster, S. Cusack, W. Petry, Dynamical transition of myoglobin revealed by inelastic neutron scattering, Nature 337 (1989) 754-756.

[5] S. Magazù, F. Migliardo, A. Benedetto, Puzzle of protein dynamical transition, J. Phys. Chem. B 115 (2011) 7736-7743.

[6] K.L. Ngai, S. Capaccioli, A. Paciaroni, Dynamics of hydrated proteins and bioprotectants: caged dynamics, b-relaxation, and a-relaxation, Biochim. Biophys. Acta 1861 (2017) 3553-3563.

[7] G. Schirò, F. Natali, A. Cupane, Physical origin of anharmonic dynamics in proteins: new insights from resolution-dependent neutron scattering on homomeric polypeptides, Phys. Rev. Lett. 109 (2012), 128102.

[8] F. Parak, E.W. Knapp, D. Kucheida, Protein dynamics. Mössbauer spectroscopy on deoxymyoglobin crystals, J. Mol. Biol. 161 (1982) 177-194.

[9] H. Frauenfelder, The Debye-Waller factor: from villain to hero in protein crystallography, Int. J. Quantum Chem. 35 (1989) 711-715.

[10] N. Engler, A. Ostermann, N. Niimura, F.G. Parak, Hydrogen atoms in proteins: positions and dynamics, Proc. Natl. Acad. Sci. U. S. A. 100 (2003) 10243-10248.

[11] A. Cupane, M. Leone, E. Vitrano, Protein dynamics - conformational disorder, vibrational coupling and anharmonicity in deoxy-hemoglobin and myoglobin, Eur. Biophys. J. 21 (1993) 385-391.

[12] M. Leone, A. Cupane, V. Militello, L. Cordone, Thernal broadening if the Soret band in heme complexes and in heme-proteins - role of iron dynamics, Eur. Biophys. J. 23 (1994) 349-352.

[13] J.B. Johnson, D.C. Lamb, H. Frauenfelder, J.D. Muller, B.H. McMahon, G.U. Nienhaus, R.D. Young, Ligand binding to hemeproteins. VI. Interconversion of taxonomic substates in carbonmonoxymyoglobin, Biophys. J. 71 (1996) 1563-1573.

[14] D.J. Bicout, G. Zaccai, Protein flexibility from the dynamical transition: a force constant analysis, Biophys. J. 80 (2001) 1115-1123.

[15] J.H. Roh, V.N. Novikov, R.B. Gregory, J.E. Curtis, Z. Chowdhuri, A.P. Sokolov, Onsets of anharmonicity in protein dynamics, Phys. Rev. Lett. 95 (2005).

[16] G. Schiro, C. Caronna, F. Natali, A. Cupane, Direct evidence of the amino acid side chain and backbone contributions to protein anharmonicity, J. Am. Chem. Soc. 132 (2010) 1371-1376.

[17] M. Fomina, G. Schiro, A. Cupane, Hydration dependence of myoglobin dynamics studied with elastic neutron scattering, differential scanning calorimetry and broadband dielectric spectroscopy, Biophys. Chem. 185 (2014) 25-31.
[18] G. Schirò, Y. Fichou, F.X. Gallat, K. Wood, F. Gabel, M. Moulin, M. Härtlein, M. Heyden, J.P. Colletier, A. Orecchini, A. Paciaroni, J. Wuttke, D.J. Tobias, M. Weik, Translational diffusion of hydration water correlates with functional motions in folded and intrinsically disordered proteins, Nat. Commun. 6 (2015) 6490

[19] G. Schirò, M. Fomina, A. Cupane, Protein dynamical transition vs. liquid-liquid phase transition in protein hydration water, J. Chem. Phys. 139 (2013) 121102.

[20] V. Conti Nibali, G. D'Angelo, A. Paciaroni, D.J. Tobias, M. Tarek, On the coupling between the collective dynamics of proteins and their hydration water, J. Phys. Chem. Lett. 5 (2014) 1181-1186.

[21] A. Paciaroni, V. Conti Nibali, A. Orecchini, C. Petrillo, M. Haertlein, M. Moulin, M. Tarek, G. D'Angelo, F. Sacchetti, Vibrational excitations of proteins and their hydration water in the far-infrared range, Chem. Phys. 424 (2013) 80-83.

[22] L. Cordone, G. Cottone, A. Cupane, A. Emanuele, S. Giuffrida, M. Levantino, Proteins in saccharides matrices and the trehalose peculiarity: biochemical and biophysical properties, Curr. Org. Chem. 19 (2015) 1684-1706.

[23] L. Cordone, G. Cottone, S. Giuffrida, F. Librizzi, Thermal evolution of the CO stretching band in carboxy-myoglobin in the light of neutron scattering data, Chem. Phys. 345 (2008) 275-282.

[24] F. Librizzi, E. Vitrano, A. Paciaroni, L. Cordone, Elastic neutron scattering of dry and rehydrated trehalose coated carboxy-myoglobin, Chem. Phys. 345 (2008) 283-288.

[25] F. Librizzi, E. Vitrano, L. Cordone, Inhibition of a substates interconversion in trehalose coated carbonmonoxy-myoglobin, in: H. Frauenfelder, G. Hummer, R. Garcia (Eds.),Biol. Phys 1999, pp. 132-138.

[26] F. Librizzi, R. Carrotta, J. Peters, A. Cupane, The effects of pressure on the energy landscape of proteins, Sci. Rep. 8 (2018)

[27] M. Erlkamp, J. Marion, N. Martinez, C. Czeslik, J. Peters, R. Winter, Influence of pressure and crowding on the sub-nanosecond dynamics of globular proteins, J. Phys. Chem. B 119 (2015) 4842-4848.

[28] N. Martinez, G. Michoud, A. Cario, J. Ollivier, B. Franzetti, M. Jebbar, P. Oger, J. Peters, High protein flexibility and reduced hydration water dynamics are key pressure adaptive strategies in prokaryotes, Sci. Rep. 6 (2016), 32816.

[29] A. Rahman, S. Singwi, A. Sjölander, Theory of slow neutron scattering by liquids. I, Phys. Rev. 126 (1962) 986-996.

[30] M. Tehei, R. Daniel, G. Zaccai, Fundamental and biotechnological applications of neutron scattering measurements for macromolecular dynamics, Eur. Biophys. J. 35 (2006) 551-558.

[31] Z. Liu, J. Huang, M. Tyagi, H. O'Neill, Q. Zhang, E. Mamontov, N. Jain, Y. Wang, J. Zhang, J.C. Smith, L. Hong, Dynamical transition of collective motions in dry proteins, Phys. Rev. Lett. 119 (2017) 48101.

[32] J. Kong, S. Yu, Fourier transform infrared spectroscopic analysis of protein secondary structures, Acta Biochim. Biophys. Sin. Shanghai 39 (2007) 549-559.

[33] A. Barth, Infrared spectroscopy of proteins, Biochim. Biophys. Acta 1767 (2007) 1073-1101.

[34] F. Piccirilli, G. Schiro, V. Vetri, S. Lupi, A. Perucchi, V. Militello, Decoding vibrational states of concanavalin A amyloid fibrils, Biophys. Chem. 199 (2015) 17-24

[35] W. Al-Azzam, E.A. Pastrana, Y. Ferrer, Q. Huang, R. Schweitzer-Stenner, K. Griebenow, Structure of poly(ethylene glycol)-modified horseradish peroxidase in organic solvents: infrared amide I spectral changes upon protein dehydration are largely caused by protein structural changes and not by water removal per se, Biophys. J. 83 (2002) 3637-3651.

[36] J.A. Rupley, G. Careri, Protein hydration and function, Adv. Protein Chem. 41 (1991) 37-172.

[37] R.M. Daniel, R.V. Dunn, J.L. Finney, J.C. Smith, The role of dynamics in enzyme activity, Annu. Rev. Biophys. Biomol. Struct. 32 (2003) 69-92.

[38] A. Paciaroni, S. Cinelli, G. Onori, Effect of the environment on the protein dynamical transition: a neutron scattering study, Biophys. J. 83 (2002) 1157-1164.

[39] G. Caliskan, D. Mechtani, J.H. Roh, A. Kisliuk, A.P. Sokolov, S. Azzam, M.T. Cicerone, S. Lin-Gibson, I. Peral, Protein and solvent dynamics: how strongly are they coupled? J. Chem. Phys. 121 (2004) 1978-1983.

[40] M.T. Cicerone, J.F. Douglas, Beta-relaxation governs protein stability in sugar-glass matrices, Soft Matter 8 (2012) 2983-2991.

[41] V.G. Sakai, S. Khodadadi, M.T. Cicerone, J.E. Curtis, A.P. Sokolov, J.H. Roh, Solvent effects on protein fast dynamics: implications for biopreservation, Soft Matter 9 (2013) 5336-5340.

[42] M. Katava, G. Stirnemann, M. Zanatta, S. Capaccioli, M. Pachetti, K.L. Ngai, F. Sterpone, A. Paciaroni, Critical structural fluctuations of proteins upon thermal unfolding challenge the Lindemann criterion, Proc. Natl. Acad. Sci. U. S. A. 114 (2017) 9361-9366.

[43] H. Jansson, R. Bergman, J. Swenson, Role of solvent for the dynamics and the glass transition of proteins, J. Phys. Chem. B 115 (2011) 4099-4109.

[44] V. Réat, G. Zaccai, M. Ferrand, C. Pfister, Functional dynamics in purple membrane, Biol. Macromol. Dyn., Adenine, Guilderland, New York 1997, pp. 117-122.

[45] V. Réat, H. Patzelt, M. Ferrand, C. Pfister, D. Oesterhelt, G. Zaccai, Dynamics of different functional parts of bacteriorhodopsin: $\mathrm{H}-\mathrm{H}-2$ labeling and neutron scattering, Proc. Natl. Acad. Sci. U. S. A. 95 (1998) 4970-4975.

[46] N.R. Zaccai, I.N. Serdyuk, G. Zaccai, Methods in Molecular Biophysics. Structure, Dynamics, Function for Biology and Medicine, Cambridge University Press, Cambridge, 2017.

[47] A.I. Lopez-Lorente, P. Wang M. Sieger, E. Vargas Catalan, M. Karlsson, F. Nikolajeff, L Österlund, B. Mizaikoff, Mid-infrared thin-film diamond waveguides combined with tunable quantum cascade lasers for analyzing the secondary structure of proteins, Phys. Status Solidi A (8) (2016) 2117-2123.

[48] H.S. Chung, A. Tokmakoff, Visualization and characterization of the infrared active amide I vibrations of proteins, J. Phys. Chem. B 110 (2006) 2888-2898.

[49] F. Demmel, W. Doster, W. Petry, A. Schulte, Vibrational frequency shifts as a probe of hydrogen bonds: thermal expansion and glass transition of myoglobin in mixed solvents, Eur. Biophys. J. 26 (1997) 327-335. 
[50] A.D. Kaposi, J. Fidy, E.S. Manas, J.M. Vanderkooi, W.W. Wright, Horseradish peroxidase monitored by infrared spectroscopy: effect of temperature, substrate and calcium, Biochim. Biophys. Acta Protein Struct. Mol. Enzymol. 1435 (1999) 41-50.

[51] E.S. Manas, Z. Getahun, W.W. Wright, W.F. Degrado, J.M. Vanderkooi, Infrared spectra of amide groups in alpha-helical proteins: evidence for hydrogen bonding between helices and water, J. Am. Chem. Soc. 122 (2000) 9883-9890.

[52] J.D. Smith, R.J. Saykally, P.L. Geissler, The effects of dissolved halide anions on hydrogen bonding in liquid water, J. Am. Chem. Soc. 129 (2007) 13847-13856.

[53] M.T. Zanni, M.C. Asplund, R.M. Hochstrasser, Two-dimensional heterodyned and stimulated infrared photon echoes of N-methylacetamide-D, J. Chem. Phys. 114 (2001) 4579-4590.

[54] T.L. Jansen, J. Knoester, A transferable electrostatic map for solvation effects on amide I vibrations and its application to linear and two-dimensional spectroscopy, J. Chem. Phys. 124 (2006).
[55] S. Hahn, S. Ham, M. Cho, Simulation studies of amide I IR absorption and twodimensional IR spectra of beta hairpins in liquid water, J. Phys. Chem. B 109 (2005) 11789-11801.

[56] B.M. Murphy, J. D'Antonio, M.C. Manning, W. Al-Azzam, Use of the amide II infrared band of proteins for secondary structure determination and comparability of higher order structure, Curr. Pharm. Biotechnol. 15 (2014) 880-889.

[57] K.E. Wilcox, E.W. Blanch, A.J. Doig, Determination of protein secondary structure from infrared spectra using partial least-squares regression, Biochemistry 55 (2016) 3794-3802.

[58] J.A. Trejo González, M.P. Longinotti, H.R. Corti, The viscosity of glycerol-water mixtures including the supercooled region, J. Chem. Eng. Data 56 (2011) 1397-1406.

[59] R.M. Daniel, J.L. Finney, V. Reat, R. Dunn, M. Ferrand, J.C. Smith, Enzyme dynamics and activity: time-scale dependence of dynamical transitions in glutamate dehydrogenase solution, Biophys. J. 77 (1999) 2184-2190. 